\title{
The CoNLL-2013 Shared Task on Grammatical Error Correction
}

\author{
Hwee Tou Ng \\ Department of Computer Science \\ National University of Singapore \\ nght d comp.nus.edu.sg
}

\author{
Siew Mei Wu \\ Centre for English Language Communication \\ National University of Singapore \\ elcwusm@nus.edu.sg
}

\author{
Yuanbin Wu and Christian Hadiwinoto \\ Department of Computer Science \\ National University of Singapore \\ $\{$ wuyb, chrhad\}@comp.nus.edu.sg \\ Joel Tetreault \\ Nuance Communications, Inc. \\ Joel. Tetreault@nuance.com
}

\begin{abstract}
The CoNLL-2013 shared task was devoted to grammatical error correction. In this paper, we give the task definition, present the data sets, and describe the evaluation metric and scorer used in the shared task. We also give an overview of the various approaches adopted by the participating teams, and present the evaluation results.
\end{abstract}

\section{Introduction}

Grammatical error correction is the shared task of the Seventeenth Conference on Computational Natural Language Learning in 2013 (CoNLL2013). In this task, given an English essay written by a learner of English as a second language, the goal is to detect and correct the grammatical errors present in the essay, and return the corrected essay.

This task has attracted much recent research interest, with two shared tasks Helping Our Own (HOO) 2011 and 2012 organized in the past two years (Dale and Kilgarriff, 2011; Dale et al., 2012). In contrast to previous CoNLL shared tasks which focused on particular subtasks of natural language processing, such as named entity recognition, semantic role labeling, dependency parsing, or coreference resolution, grammatical error correction aims at building a complete end-to-end application. This task is challenging since for many error types, current grammatical error correction systems do not achieve high performance and much research is still needed. Also, tackling this task has far-reaching impact, since it is estimated that hundreds of millions of people worldwide are learning English and they benefit directly from an automated grammar checker.
The CoNLL-2013 shared task provides a forum for participating teams to work on the same grammatical error correction task, with evaluation on the same blind test set using the same evaluation metric and scorer. This overview paper contains a detailed description of the shared task, and is organized as follows. Section 2 provides the task definition. Section 3 describes the annotated training data provided and the blind test data. Section 4 describes the evaluation metric and the scorer. Section 5 lists the participating teams and outlines the approaches to grammatical error correction used by the teams. Section 6 presents the results of the shared task. Section 7 concludes the paper.

\section{Task Definition}

The goal of the CoNLL-2013 shared task is to evaluate algorithms and systems for automatically detecting and correcting grammatical errors present in English essays written by second language learners of English. Each participating team is given training data manually annotated with corrections of grammatical errors. The test data consists of new, blind test essays. Preprocessed test essays, which have been sentencesegmented and tokenized, are also made available to the participating teams. Each team is to submit its system output consisting of the automatically corrected essays, in sentence-segmented and tokenized form.

Grammatical errors consist of many different types, including articles or determiners, prepositions, noun form, verb form, subject-verb agreement, pronouns, word choice, sentence structure, punctuation, capitalization, etc. Of all the error types, determiners and prepositions are among 
the most frequent errors made by learners of English. Not surprisingly, much published research on grammatical error correction focuses on article and preposition errors (Han et al., 2006; Gamon, 2010; Rozovskaya and Roth, 2010; Tetreault et al., 2010; Dahlmeier and $\mathrm{Ng}, 2011 \mathrm{~b}$ ), with relatively less work on correcting word choice errors (Dahlmeier and Ng, 2011a). Article and preposition errors were also the only error types featured in the HOO 2012 shared task. Likewise, although all error types were included in the HOO 2011 shared task, almost all participating teams dealt with article and preposition errors only (besides spelling and punctuation errors).

In the CoNLL-2013 shared task, it was felt that the community is now ready to deal with more error types, including noun number, verb form, and subject-verb agreement, besides articles/determiners and prepositions. Table 1 shows examples of the five error types in our shared task.

Since there are five error types in our shared task compared to two in HOO 2012, there is a greater chance of encountering multiple, interacting errors in a sentence in our shared task. This increases the complexity of our shared task relative to that of HOO 2012. To illustrate, consider the following sentence:
Although we have to admit some bad effect which is brought by the new technology, still the advantages of the new technologies cannot be simply dis- carded.

The noun number error effect needs to be corrected (effect $\rightarrow$ effects). This necessitates the correction of a subject-verb agreement error (is $\rightarrow$ are). A pipeline system in which corrections for subjectverb agreement errors occur strictly before corrections for noun number errors would not be able to arrive at a fully corrected sentence for this example. The ability to correct multiple, interacting errors is thus necessary in our shared task. The recent work of (Dahlmeier and Ng, 2012a), for example, is designed to deal with multiple, interacting errors.

Note that the essays in the training data and the test essays naturally contain grammatical errors of all types, beyond the five error types focused in our shared task. In the automatically corrected essays returned by a participating system, only corrections necessary to correct errors of the five types are made. The other errors are to be left uncorrected.

\section{Data}

This section describes the training and test data released to each participating team in our shared task.

\subsection{Training Data}

The training data provided in our shared task is the NUCLE corpus, the NUS Corpus of Learner English (Dahlmeier et al., 2013). As noted by (Leacock et al., 2010), the lack of a manually annotated and corrected corpus of English learner texts has been an impediment to progress in grammatical error correction, since it prevents comparative evaluations on a common benchmark test data set. NUCLE was created precisely to fill this void. It is a collection of 1,414 essays written by students at the National University of Singapore (NUS) who are non-native speakers of English. The essays were written in response to some prompts, and they cover a wide range of topics, such as environmental pollution, health care, etc. The grammatical errors in these essays have been hand-corrected by professional English instructors at NUS. For each grammatical error instance, the start and end character offsets of the erroneous text span are marked, and the error type and the correction string are provided. Manual annotation is carried out using a graphical user interface specifically built for this purpose. The error annotations are saved as stand-off annotations, in SGML format.

To illustrate, consider the following sentence at the start of the first paragraph of an essay:

From past to the present, many important innovations have surfaced.

There is an article/determiner error (past $\rightarrow$ the past) in this sentence. The error annotation, also called correction or edit, in SGML format is shown in Figure 1. start_par (end_par) denotes the paragraph ID of the start (end) of the erroneous text span (paragraph ID starts from 0 by convention). start_off (end_off) denotes the character offset of the start (end) of the erroneous text span (again, character offset starts from 0 by convention). The error tag is ArtOrDet, and the correction string is the past. 


\begin{tabular}{|l|l|l|l|}
\hline Error tag & \multicolumn{1}{|c|}{ Error type } & \multicolumn{1}{|c|}{ Example sentence } & \multicolumn{1}{c|}{ Correction (edit) } \\
\hline ArtOrDet & Article or determiner & $\begin{array}{l}\text { In late nineteenth century, there } \\
\text { was a severe air crash happening } \\
\text { at Miami international airport. }\end{array}$ & late $\rightarrow$ the late \\
\hline Prep & Preposition & $\begin{array}{l}\text { Also tracking people is very } \\
\text { dangerous if it has been con- } \\
\text { trolled by bad men in a not good } \\
\text { purpose. }\end{array}$ & in $\rightarrow$ for \\
\hline Nn & Noun number & $\begin{array}{l}\text { I think such powerful device } \\
\text { shall not be made easily avail- } \\
\text { able. }\end{array}$ & device $\rightarrow$ devices \\
\hline Vform & Verb form & $\begin{array}{l}\text { However, it is an achievement as } \\
\text { it is an indication that our soci- } \\
\text { ety is progressed well and peo- } \\
\text { ple are living in better condi- } \\
\text { tions. }\end{array}$ & progressed $\rightarrow$ progressing \\
\hline SVA & Subject-verb agreement & $\begin{array}{l}\text { People still prefers to bear the } \\
\text { risk and allow their pets to have } \\
\text { maximum freedom. }\end{array}$ & prefers $\rightarrow$ prefer \\
\hline
\end{tabular}

Table 1: The five error types in our shared task.

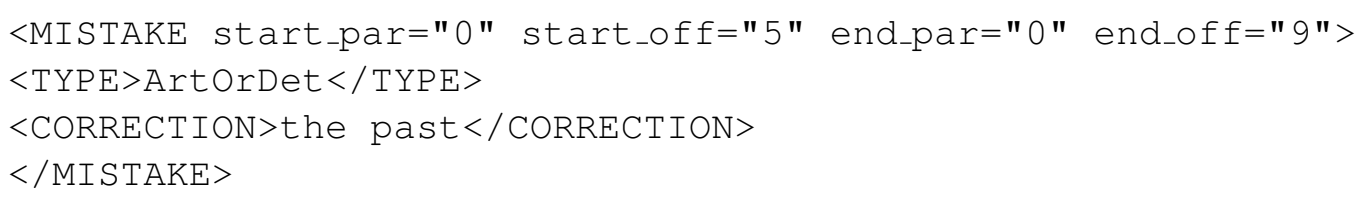

Figure 1: An example error annotation.

The NUCLE corpus was first used in (Dahlmeier and Ng, 2011b), and has been publicly available for research purposes since June $2011^{1}$. All instances of grammatical errors are annotated in NUCLE, and the errors are classified into 27 error types (Dahlmeier et al., 2013).

To help participating teams in their preparation for the shared task, we also performed automatic preprocessing of the NUCLE corpus and released the preprocessed form of NUCLE. The preprocessing operations performed on the NUCLE essays include sentence segmentation and word tokenization using the NLTK toolkit (Bird et al., 2009), and part-of-speech (POS) tagging, constituency and dependency tree parsing using the Stanford parser (Klein and Manning, 2003; de Marneffe et al., 2006). The error annotations, which are originally at the character level, are then mapped to error annotations at the word token level. Error annotations at the word token

\footnotetext{
${ }^{1}$ http://www.comp.nus.edu.sg/ nlp/corpora.html
}

level also facilitate scoring, as we will see in Section 4 , since our scorer operates by matching tokens. Note that although we released our own preprocessed version of NUCLE, the participating teams were however free to perform their own preprocessing if they so preferred.

\subsubsection{Revised version of NUCLE}

NUCLE release version 2.3 was used in the CoNLL-2013 shared task. In this version, 17 essays were removed from the first release of NUCLE since these essays were duplicates with multiple annotations.

In the original NUCLE corpus, there is not an explicit preposition error type. Instead, preposition errors are part of the Wcip (wrong collocation/idiom/preposition) and Rloc (local redundancy) error types. The Wcip error type combines errors concerning collocations, idioms, and prepositions together into one error type. The Rloc error type annotates extraneous words which are redundant and should be removed, and they include redundant articles, determiners, and prepositions. 


\begin{tabular}{|l|r|r|}
\hline & $\begin{array}{c}\text { Training data } \\
\text { (NUCLE) }\end{array}$ & Test data \\
\hline \# essays & 1,397 & 50 \\
\hline \# sentences & 57,151 & 1,381 \\
\hline \# word tokens & $1,161,567$ & 29,207 \\
\hline
\end{tabular}

Table 2: Statistics of training and test data.

In our shared task, in order to facilitate the detection and correction of article/determiner errors and preposition errors, we performed automatic mapping of error types in the original NUCLE corpus. The mapping relies on POS tags, constituent parse trees, and error annotations at the word token level. Specifically, we map the error types Wcip and Rloc to Prep, Wci, ArtOrDet, and Rloc-. Prepositions in the error type Wcip or Rloc are mapped to a new error type Prep, and redundant articles or determiners in the error type Rloc are mapped to ArtOrDet. The remaining unaffected Wcip errors are assigned the new error type Wci and the remaining unaffected Rloc errors are assigned the new error type Rloc-. The code that performs automatic error type mapping was also provided to the participating teams.

The statistics of the NUCLE corpus (release 2.3 version) are shown in Table 2. The distribution of errors among the five error types is shown in Table 3. The newly added noun number error type in our shared task accounts for the second highest number of errors among the five error types. The five error types in our shared task constitute $35 \%$ of all grammatical errors in the training data, and $47 \%$ of all errors in the test data. These figures support our choice of these five error types to be the focus of our shared task, since they account for a large percentage of all grammatical errors in English learner essays.

While the NUCLE corpus is provided in our shared task, participating teams are free to not use NUCLE, or to use additional resources and tools in building their grammatical error correction systems, as long as these resources and tools are publicly available and not proprietary. For example, participating teams are free to use the Cambridge FCE corpus (Yannakoudakis et al., 2011; Nicholls, 2003) (the training data provided in HOO 2012 (Dale et al., 2012)) as additional training data.

\begin{tabular}{|l|r|r|r|r|}
\hline Error tag & $\begin{array}{c}\text { Training } \\
\text { data } \\
\text { (NUCLE) }\end{array}$ & \multicolumn{1}{c|}{$\begin{array}{c}\text { Test } \\
\text { data }\end{array}$} & \multicolumn{1}{|c|}{} \\
\hline ArtOrDet & 6,658 & 14.8 & 690 & 19.9 \\
\hline Prep & 2,404 & 5.3 & 312 & 9.0 \\
\hline Nn & 3,779 & 8.4 & 396 & 11.4 \\
\hline Vform & 1,453 & 3.2 & 122 & 3.5 \\
\hline SVA & 1,527 & 3.4 & 124 & 3.6 \\
\hline 5 types & 15,821 & 35.1 & 1,644 & 47.4 \\
\hline all types & 45,106 & 100.0 & 3,470 & 100.0 \\
\hline
\end{tabular}

Table 3: Error type distribution of the training and test data.

\subsection{Test Data}

25 NUS students, who are non-native speakers of English, were recruited to write new essays to be used as blind test data in the shared task. Each student wrote two essays in response to the two prompts shown in Table 4, one essay per prompt. Essays written using the first prompt are present in the NUCLE training data, while the second prompt is a new prompt not used previously. As a result, 50 test essays were collected. The statistics of the test essays are shown in Table 2.

Error annotation on the test essays was carried out by a native speaker of English who is a lecturer at the NUS Centre for English Language Communication. The distribution of errors in the test essays among the five error types is shown in Table 3. The test essays were then preprocessed in the same manner as the NUCLE corpus. The preprocessed test essays were released to the participating teams.

Unlike the test data used in HOO 2012 which was proprietary and not available after the shared task, the test essays and their error annotations in the CoNLL-2013 shared task are freely available after the shared task.

\section{Evaluation Metric and Scorer}

A grammatical error correction system is evaluated by how well its proposed corrections or edits match the gold-standard edits. An essay is first sentence-segmented and tokenized before evaluation is carried out on the essay. To illustrate, consider the following tokenized sentence $S$ written by an English learner:

There is no a doubt, tracking system 


\begin{tabular}{|c|l|}
\hline ID & \multicolumn{1}{|c|}{ Prompt } \\
\hline 1 & $\begin{array}{l}\text { Surveillance technology such as RFID (radio-frequency identification) should not be used to } \\
\text { track people (e.g., human implants and RFID tags on people or products). Do you agree? Sup- } \\
\text { port your argument with concrete examples. }\end{array}$ \\
\hline 2 & $\begin{array}{l}\text { Population aging is a global phenomenon. Studies have shown that the current average life span } \\
\text { is over 65. Projections of the United Nations indicate that the population aged } 60 \text { or over in } \\
\text { developed and developing countries is increasing at 2\% to 3\% annually. Explain why rising life } \\
\text { expectancies can be considered both a challenge and an achievement. }\end{array}$ \\
\hline
\end{tabular}

Table 4: The two prompts used for the test essays.

has brought many benefits in this information age .

The set of gold-standard edits of a human annotator is $\mathbf{g}=\{$ a doubt $\rightarrow$ doubt, system $\rightarrow$ systems, has $\rightarrow$ have $\}$. Suppose the tokenized output sentence $H$ of a grammatical error correction system given the above sentence is:

There is no doubt, tracking system has brought many benefits in this information age .

That is, the set of system edits is $\mathbf{e}=\{\mathbf{a}$ doubt $\rightarrow$ doubt $\}$. The performance of the grammatical error correction system is measured by how well the two sets $\mathbf{g}$ and $\mathbf{e}$ match, in the form of recall $R$, precision $P$, and $F_{1}$ measure: $R=1 / 3, P=$ $1 / 1, F_{1}=2 R P /(R+P)=1 / 2$.

More generally, given a set of $n$ sentences, where $\mathbf{g}_{i}$ is the set of gold-standard edits for sentence $i$, and $\mathbf{e}_{i}$ is the set of system edits for sentence $i$, recall, precision, and $F_{1}$ are defined as follows:

$$
\begin{gathered}
R=\frac{\sum_{i=1}^{n}\left|\mathbf{g}_{i} \cap \mathbf{e}_{i}\right|}{\sum_{i=1}^{n}\left|\mathbf{g}_{i}\right|} \\
P=\frac{\sum_{i=1}^{n}\left|\mathbf{g}_{i} \cap \mathbf{e}_{i}\right|}{\sum_{i=1}^{n}\left|\mathbf{e}_{i}\right|} \\
F_{1}=\frac{2 \times R \times P}{R+P}
\end{gathered}
$$

where the intersection between $\mathbf{g}_{i}$ and $\mathbf{e}_{i}$ for sentence $i$ is defined as

$$
\mathbf{g}_{i} \cap \mathbf{e}_{i}=\left\{e \in \mathbf{e}_{i} \mid \exists g \in \mathbf{g}_{i}, \operatorname{match}(g, e)\right\}
$$

Evaluation by the HOO scorer (Dale and Kilgarriff, 2011) is based on computing recall, precision, and $F_{1}$ measure as defined above.

Note that there are multiple ways to specify a set of gold-standard edits that denote the same corrections. For example, in the above learner-written sentence $S$, alternative but equivalent sets of goldstandard edits are $\{a \rightarrow \epsilon$, system $\rightarrow$ systems, has $\rightarrow$ have $\},\{a \rightarrow \epsilon$, system has $\rightarrow$ systems have $\}$, etc. Given the same learner-written sentence $S$ and the same system output sentence $H$ shown above, one would expect a scorer to give the same $R, P, F_{1}$ scores regardless of which of the equivalent sets of gold-standard edits is specified by an annotator.

However, this is not the case with the $\mathrm{HOO}$ scorer. This is because the HOO scorer uses GNU wdiff $f^{2}$ to extract the differences between the learner-written sentence $S$ and the system output sentence $H$ to form a set of system edits. Since in general there are multiple ways to specify a set of gold-standard edits that denote the same corrections, the set of system edits computed by the HOO scorer may not match the set of gold-standard edits specified, leading to erroneous scores. In the above example, the set of system edits computed by the HOO scorer for $S$ and $H$ is $\{\mathrm{a} \rightarrow \epsilon\}$. Given that the set of gold-standard edits $\mathrm{g}$ is $\{$ a doubt $\rightarrow$ doubt, system $\rightarrow$ systems, has $\rightarrow$ have , the scores computed by the HOO scorer are $R=P=F_{1}=0$, which are erroneous.

The MaxMatch $\left(\mathrm{M}^{2}\right)$ scorer $^{3}$ (Dahlmeier and $\mathrm{Ng}, 2012 \mathrm{~b}$ ) was designed to overcome this limitation of the HOO scorer. The key idea is that the set of system edits automatically computed and used in scoring should be the set that maximally matches the set of gold-standard edits specified by the annotator. The $\mathrm{M}^{2}$ scorer uses an efficient algorithm to search for such a set of system edits using an edit lattice. In the above example, given $S, H$, and $\mathrm{g}$, the $\mathrm{M}^{2}$ scorer is able to come up with the best matching set of system edits $\mathbf{e}=\{\mathrm{a}$ doubt $\rightarrow$ doubt $\}$, thus giving the correct scores $R=1 / 3, P=1 / 1, F_{1}=1 / 2$. We use the $\mathbf{M}^{2}$

\footnotetext{
${ }^{2} \mathrm{http} / / /$ www.gnu.org/s/wdiff/

${ }^{3}$ http://www.comp.nus.edu.sg/ nlp/software.html
} 
scorer in the CoNLL-2013 shared task.

The original $\mathrm{M}^{2}$ scorer implemented in (Dahlmeier and $\mathrm{Ng}, 2012 \mathrm{~b}$ ) assumes that there is one set of gold-standard edits $\mathbf{g}_{i}$ for each sentence $i$. However, it is often the case that multiple alternative corrections are acceptable for a sentence. As we allow participating teams to submit alternative sets of gold-standard edits for a sentence, we also extend the $\mathrm{M}^{2}$ scorer to deal with multiple alternative sets of gold-standard edits.

Based on Equations 1 and 2, Equation 3 can be re-expressed as:

$$
F_{1}=\frac{2 \times \sum_{i=1}^{n}\left|\mathbf{g}_{i} \cap \mathbf{e}_{i}\right|}{\sum_{i=1}^{n}\left(\left|\mathbf{g}_{i}\right|+\left|\mathbf{e}_{i}\right|\right)}
$$

To deal with multiple alternative sets of goldstandard edits $\mathbf{g}_{i}$ for a sentence $i$, the extended $\mathrm{M}^{2}$ scorer chooses the $\mathrm{g}_{i}$ that maximizes the cumulative $F_{1}$ score for sentences $1, \ldots, i$. Ties are broken based on the following criteria: first choose the $\mathrm{g}_{i}$ that maximizes the numerator $\sum_{i=1}^{n}\left|\mathbf{g}_{i} \cap \mathbf{e}_{i}\right|$, then choose the $\mathbf{g}_{i}$ that minimizes the denominator $\sum_{i=1}^{n}\left(\left|\mathbf{g}_{i}\right|+\left|\mathbf{e}_{i}\right|\right)$, finally choose the $\mathbf{g}_{i}$ that appears first in the list of alternatives.

\section{Approaches}

54 teams registered to participate in the shared task, out of which 17 teams submitted the output of their grammatical error correction systems by the deadline. These teams are listed in Table 5. Each team is assigned a 3 to 4-letter team ID. In the remainder of this paper, we will use the assigned team ID to refer to a participating team. Every team submitted a system description paper (the only exception is the SJT2 team).

Many different approaches are adopted by participating teams in the CoNLL-2013 shared task, and Table 6 summarizes these approaches. A commonly used approach in the shared task and in grammatical error correction research in general is to build a classifier for each error type. For example, the classifier for noun number returns the classes $\{$ singular, plural $\}$, the classifier for article returns the classes $\{\mathrm{a} / \mathrm{an}$, the, $\epsilon\}$, etc. The classifier for an error type may be learned from training examples encoding the surrounding context of an error occurrence, or may be specified by deterministic hand-crafted rules, or may be built using a hybrid approach combining both machine learning and hand-crafted rules. These approaches are denoted by M, R, and $\mathrm{H}$ respectively in Table 6 .

The machine translation approach (denoted by $\mathrm{T}$ in Table 6) to grammatical error correction treats the task as "translation" from bad English to good English. Both phrase-based translation and syntax-based translation approaches are used by teams in the CoNLL-2013 shared task. Another related approach is the language modeling approach (denoted by $\mathrm{L}$ in Table 6), in which the probability of a learner sentence is compared with the probability of a candidate corrected sentence, based on a language model built from a background corpus. The candidate correction is chosen if it results in a corrected sentence with a higher probability. In general, these approaches are not mutually exclusive. For example, the work of (Dahlmeier and Ng, 2012a; Yoshimoto et al., 2013) includes elements of machine learningbased classification, machine translation, and language modeling approaches.

When different approaches are used to tackle different error types by a system, we break down the error types into different rows in Table 6, and specify the approach used for each group of error types. For instance, the HIT team uses a machine learning approach to deal with article/determiner, noun number, and preposition errors, and a rulebased approach to deal with subject-verb agreement and verb form errors. As such, the entry for HIT is sub-divided into two rows, to make it clear which particular error type is handled by which approach.

Table 6 also shows the linguistic features used by the participating teams, which include lexical features (i.e., words, collocations, n-grams), partsof-speech (POS), constituency parses, dependency parses, and semantic features (including semantic role labels).

While all teams in the shared task use the NUCLE corpus, they are also allowed to use additional external resources (both corpora and tools) so long as they are publicly available and not proprietary. The external resources used by the teams are also listed in Table 6.

\section{Results}

All submitted system output was evaluated using the $\mathrm{M}^{2}$ scorer, based on the error annotations provided by our annotator. The recall $(R)$, precision $(P)$, and $F_{1}$ measure of all teams are shown in Table 7. The performance of the teams varies greatly, 


\begin{tabular}{|l|l|}
\hline Team ID & \multicolumn{1}{|c|}{ Affiliation } \\
\hline CAMB & University of Cambridge \\
\hline HIT & Harbin Institute of Technology \\
\hline IITB & Indian Institute of Technology, Bombay \\
\hline KOR & Korea University \\
\hline NARA & Nara Institute of Science and Technology \\
\hline NTHU & National Tsing Hua University \\
\hline SAAR & Saarland University \\
\hline SJT1 & Shanghai Jiao Tong University (Team \#1) \\
\hline SJT2 & Shanghai Jiao Tong University (Team \#2) \\
\hline STAN & Stanford University \\
\hline STEL & Stellenbosch University \\
\hline SZEG & University of Szeged \\
\hline TILB & Tilburg University \\
\hline TOR & University of Toronto \\
\hline UAB & Universitat Autònoma de Barcelona \\
\hline UIUC & University of Illinois at Urbana-Champaign \\
\hline UMC & University of Macau \\
\hline
\end{tabular}

Table 5: The list of 17 participating teams.

\begin{tabular}{|c|l|r|r|r|}
\hline Rank & Team & $\mathbf{R}$ & \multicolumn{1}{c|}{$\mathbf{P}$} & $\mathbf{F}_{1}$ \\
\hline 1 & UIUC & 23.49 & 46.45 & 31.20 \\
\hline 2 & NTHU & 26.35 & 23.80 & 25.01 \\
\hline 3 & HIT & 16.56 & 35.65 & 22.61 \\
\hline 4 & NARA & 18.62 & 27.39 & 22.17 \\
\hline 5 & UMC & 17.53 & 28.49 & 21.70 \\
\hline 6 & STEL & 13.33 & 27.00 & 17.85 \\
\hline 7 & SJT1 & 10.96 & 40.18 & 17.22 \\
\hline 8 & CAMB & 10.10 & 39.15 & 16.06 \\
\hline 9 & IITB & 4.99 & 28.18 & 8.48 \\
\hline 10 & STAN & 4.69 & 25.50 & 7.92 \\
\hline 11 & TOR & 4.81 & 17.67 & 7.56 \\
\hline 12 & KOR & 3.71 & 43.88 & 6.85 \\
\hline 13 & TILB & 7.24 & 6.25 & 6.71 \\
\hline 14 & SZEG & 3.16 & 5.52 & 4.02 \\
\hline 15 & UAB & 1.22 & 12.42 & 2.22 \\
\hline 16 & SAAR & 1.10 & 27.69 & 2.11 \\
\hline 17 & SJT2 & 0.24 & 13.33 & 0.48 \\
\hline
\end{tabular}

Table 7: Scores (in \%) without alternative answers.

from barely half a per cent to $31.20 \%$ for the top team.

The nature of grammatical error correction is such that multiple, different corrections are often acceptable. In order to allow the participating teams to raise their disagreement with the original gold-standard annotations provided by the annotator, and not understate the performance of the teams, we allow the teams to submit their proposed alternative answers. This was also the practice adopted in HOO 2011 and HOO 2012. Specifically, after the teams submitted their system output and the error annotations on the test essays were released, we allowed the teams to propose alternative answers (gold-standard edits), to be submitted within four days after the initial error annotations were released. The same annotator who provided the error annotations on the test essays also judged the alternative answers proposed by the teams, to ensure consistency. In all, five teams (NTHU, STEL, TOR, UIUC, UMC) submitted alternative answers.

The same submitted system output was then evaluated using the extended $\mathrm{M}^{2}$ scorer, with the original annotations augmented with the alternative answers. Table 8 shows the recall $(R)$, precision $(P)$, and $F_{1}$ measure of all teams under this new evaluation setting.

The $F_{1}$ measure of every team improves when 


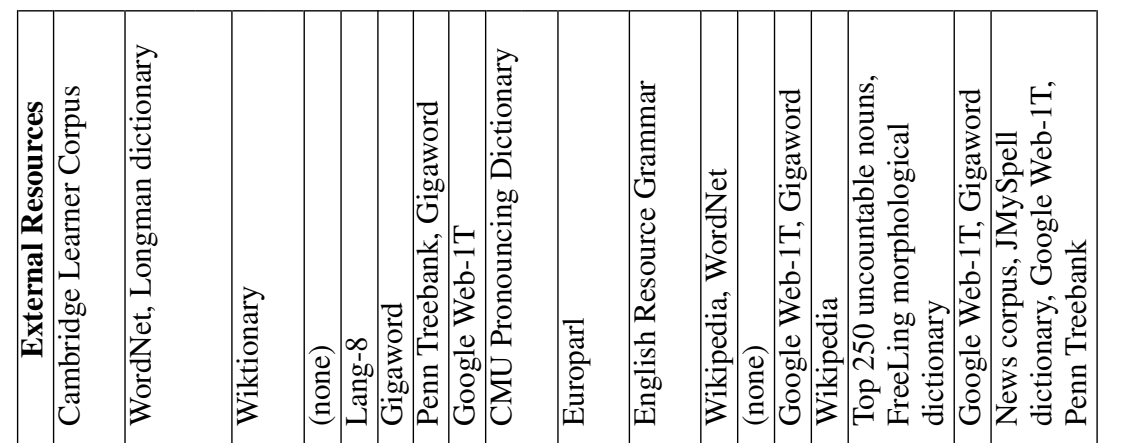

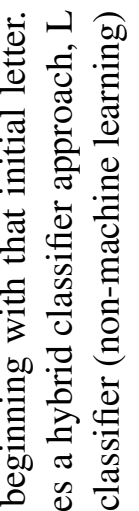

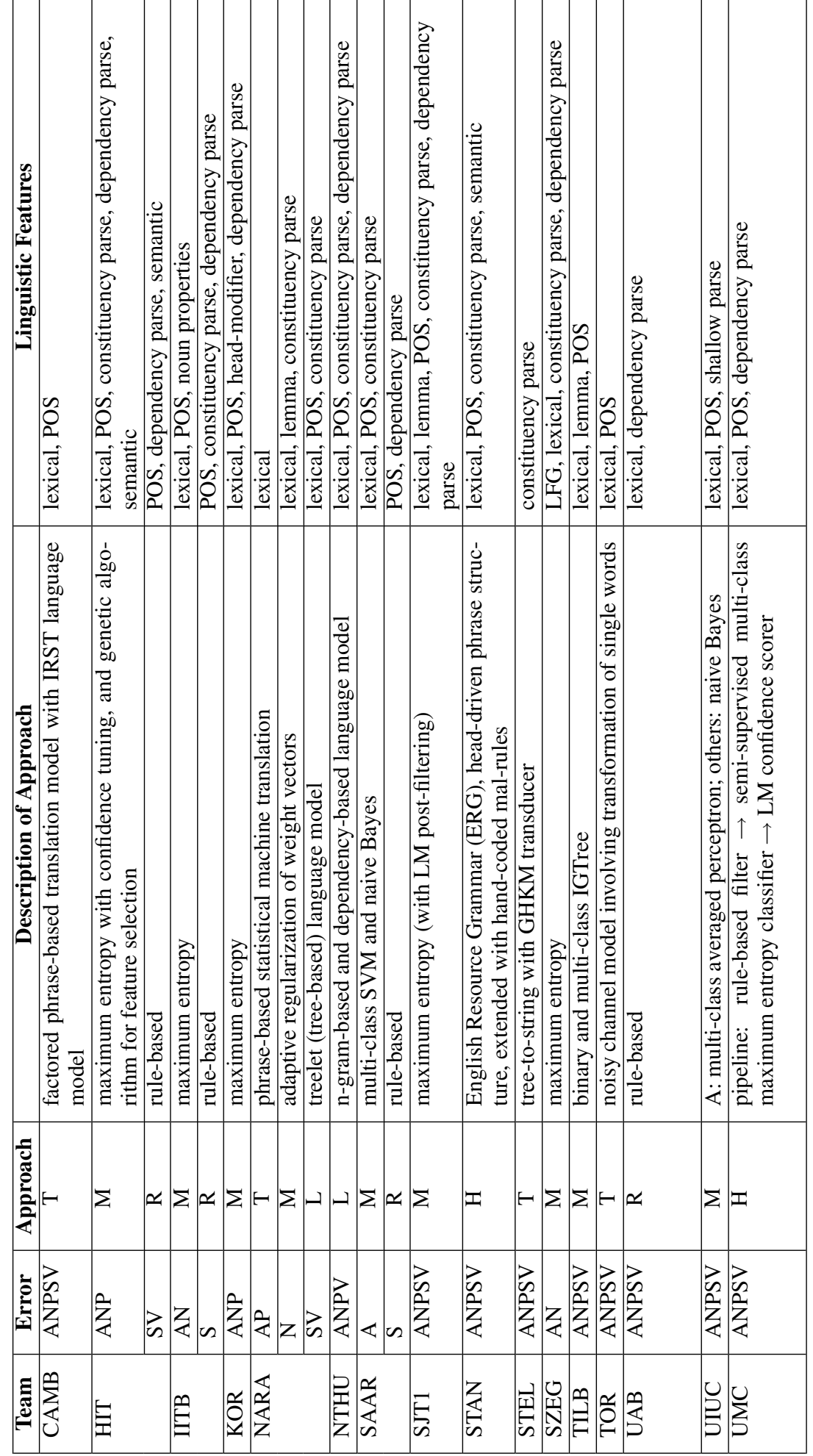

융

过

可说

\&

过

च

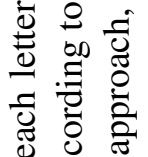

过

运

हี 0

능

ㅎํㅇ

ปั

寻

合

हี

吾

0 ष

흐웡

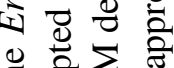

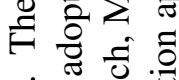

$\dot{\theta} \frac{0}{0}$ :

む

bo 言 ठ

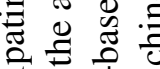

.

艺 $\frac{2}{n}$

छี 음

응

过

2 증

$\because$ 造造

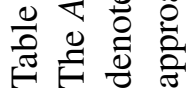


evaluated with alternative answers. Not surprisingly, the teams which submitted alternative answers tend to show the greatest improvements in their $F_{1}$ measure. Overall, the UIUC team (Rozovskaya et al., 2013) achieves the best $F_{1}$ measure, with a clear lead over the other teams in the shared task, under both evaluation settings (without and with alternative answers).

For future research which uses the test data of the CoNLL-2013 shared task, we recommend that evaluation be carried out in the setting that does not use alternative answers, to ensure a fairer evaluation. This is because the scores of the teams which submitted alternative answers tend to be higher in a biased way when evaluated with alternative answers.

\begin{tabular}{|c|l|r|r|r|}
\hline Rank & Team & $\mathbf{R}$ & \multicolumn{1}{|c|}{$\mathbf{P}$} & $\mathbf{F}_{1}$ \\
\hline 1 & UIUC & 31.87 & 62.19 & 42.14 \\
\hline 2 & NTHU & 34.62 & 30.57 & 32.46 \\
\hline 3 & UMC & 23.66 & 37.12 & 28.90 \\
\hline 4 & NARA & 24.05 & 33.92 & 28.14 \\
\hline 5 & HIT & 20.29 & 41.75 & 27.31 \\
\hline 6 & STEL & 18.91 & 37.12 & 25.05 \\
\hline 7 & CAMB & 14.19 & 52.11 & 22.30 \\
\hline 8 & SJT1 & 13.67 & 47.77 & 21.25 \\
\hline 9 & TOR & 8.77 & 30.67 & 13.64 \\
\hline 10 & IITB & 6.55 & 34.93 & 11.03 \\
\hline 11 & STAN & 5.86 & 29.93 & 9.81 \\
\hline 12 & KOR & 4.78 & 53.24 & 8.77 \\
\hline 13 & TILB & 9.29 & 7.60 & 8.36 \\
\hline 14 & SZEG & 4.07 & 6.67 & 5.06 \\
\hline 15 & UAB & 1.81 & 17.39 & 3.28 \\
\hline 16 & SAAR & 1.68 & 40.00 & 3.23 \\
\hline 17 & SJT2 & 0.33 & 16.67 & 0.64 \\
\hline
\end{tabular}

Table 8: Scores (in \%) with alternative answers.

We are also interested in the analysis of scores of each of the five error types. To compute the recall of an error type, we need to know the error type of each gold-standard edit, which is provided by the annotator. To compute the precision of each error type, we need to know the error type of each system edit, which however is not available since the submitted system output only contains the corrected sentences with no indication of the error type of the system edits.

In order to determine the error type of system edits, we first perform POS tagging on the submitted system output using the Stanford parser (Klein and Manning, 2003). We also make use of the POS tags assigned in the preprocessed form of the test essays. We then assign an error type to a system edit based on the automatically determined POS tags, as follows:

- ArtOrDet: The system edit involves a change (insertion, deletion, or substitution) of words tagged as article/determiner, i.e., DT or PDT.

- Prep: The system edit involves a change of words tagged as preposition, i.e., IN or TO.

- Nn: The system edit involves a change of words such that a word in the source string is a singular noun (tagged as NN or NNP) and a word in the replacement string is a plural noun (tagged as NNS or NNPS), or vice versa. Since a word tagged as JJ (adjective) can serve as a noun, a system edit that involves a change of POS tags from JJ to one of $\{\mathrm{NN}, \mathrm{NNP}, \mathrm{NNS}, \mathrm{NNPS}\}$ or vice versa also qualifies.

- Vform/SVA: The system edit involves a change of words tagged as one of the verb POS tags, i.e., VB, VBD, VBG, VBN, VBP, and VBZ.

The verb form and subject-verb agreement error types are grouped together into one category, since it is difficult to automatically distinguish the two in a reliable way.

The scores when distinguished by error type are shown in Tables 9 and 10. Based on the $F_{1}$ measure of each error type, the noun number error type gives the highest scores, and preposition errors remain the most challenging error type to correct.

\section{Conclusions}

The CoNLL-2013 shared task saw the participation of 17 teams worldwide to evaluate their grammatical error correction systems on a common test set, using a common evaluation metric and scorer. The five error types included in the shared task account for at least one-third to close to one-half of all errors in English learners' essays. The best system in the shared task achieves an $F_{1}$ score of $42 \%$, when it is scored with multiple acceptable answers. There is still much room for improvement, both in the accuracy of grammatical error correction systems, and in the coverage of systems to deal with a more comprehensive set of error 


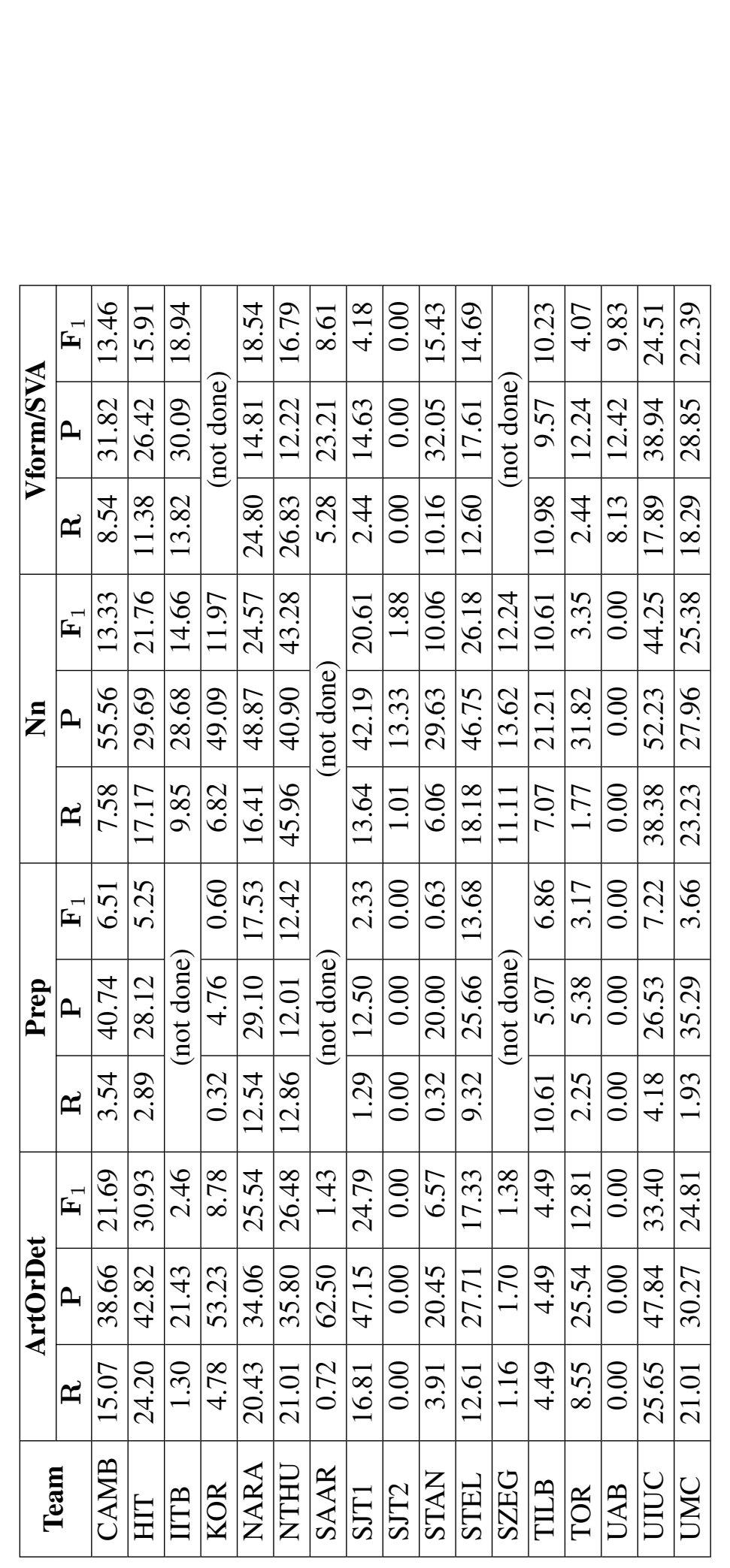

芯

$\stackrel{\infty}{=}$

$\sum^{0}$

कृ

$\frac{\bar{\Xi}}{3}$

ฮ

흘

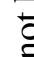

\&

离

会

䒕

్ㅗ

苂

.

ฐ

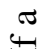

ฏ

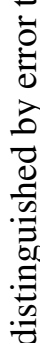

这

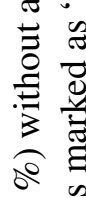

$\Xi$

¿

次

aิ

훙 


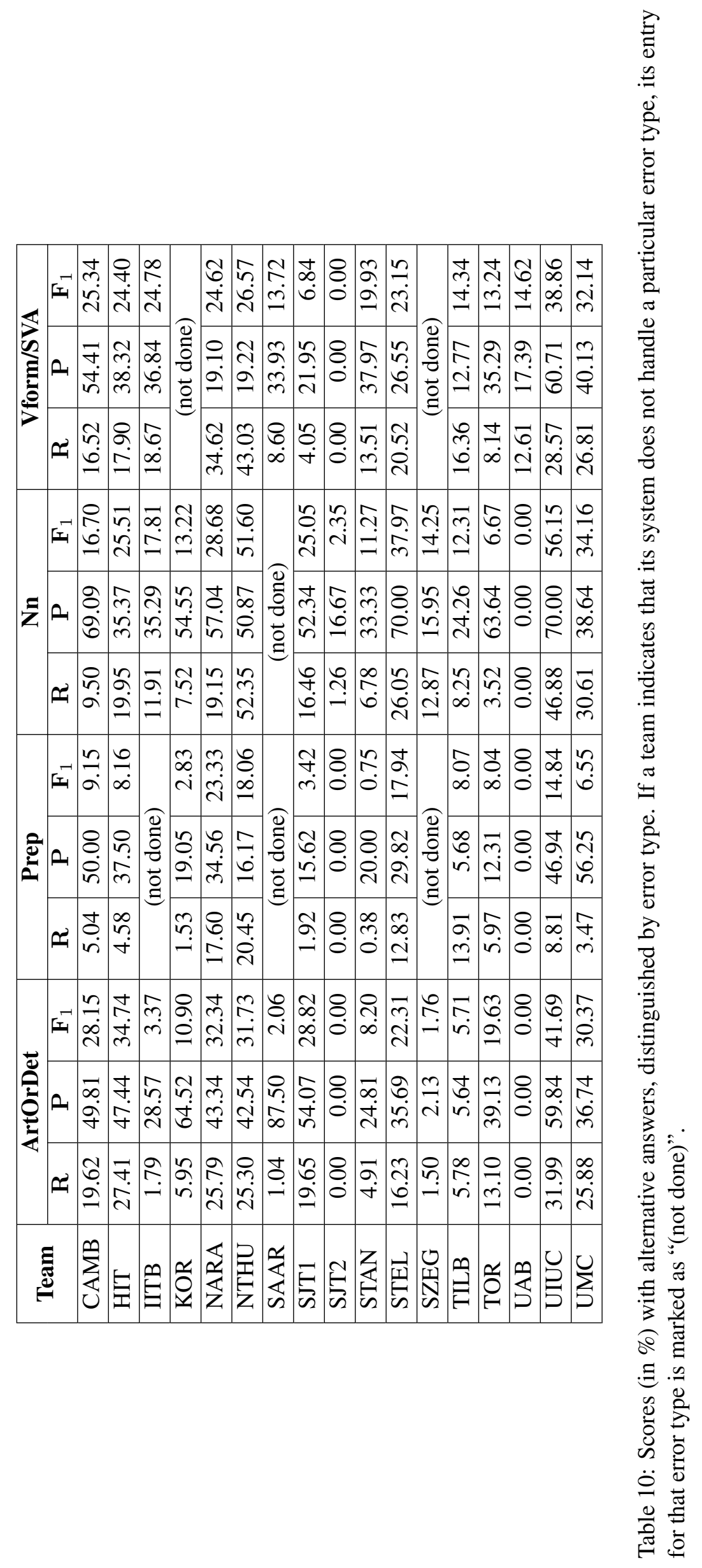


types. The evaluation data sets and scorer used in our shared task serve as a benchmark for future research on grammatical error correction ${ }^{4}$.

\section{Acknowledgments}

This research is supported by the Singapore National Research Foundation under its International Research Centre@Singapore Funding Initiative and administered by the IDM Programme Office.

\section{References}

Steven Bird, Ewan Klein, and Edward Loper. 2009. Natural Language Processing with Python. O'Reilly Media.

Daniel Dahlmeier and Hwee Tou Ng. 2011a. Correcting semantic collocation errors with L1-induced paraphrases. In Proceedings of the 2011 Conference on Empirical Methods in Natural Language Processing, pages 107-117.

Daniel Dahlmeier and Hwee Tou Ng. 2011b. Grammatical error correction with alternating structure optimization. In Proceedings of the 49th Annual Meeting of the Association for Computational Linguistics, pages 915-923.

Daniel Dahlmeier and Hwee Tou Ng. 2012a. A beamsearch decoder for grammatical error correction. In Proceedings of the 2012 Joint Conference on Empirical Methods in Natural Language Processing and Computational Natural Language Learning, pages 568-578.

Daniel Dahlmeier and Hwee Tou Ng. 2012b. Better evaluation for grammatical error correction. In Proceedings of the 2012 Conference of the North American Chapter of the Association for Computational Linguistics: Human Language Technologies, pages 568-572.

Daniel Dahlmeier, Hwee Tou Ng, and Siew Mei Wu. 2013. Building a large annotated corpus of learner English: The NUS Corpus of Learner English. In Proceedings of the Eighth Workshop on Innovative Use of NLP for Building Educational Applications, pages 22-31.

Robert Dale and Adam Kilgarriff. 2011. Helping Our Own: The HOO 2011 pilot shared task. In Proceedings of the 13th European Workshop on Natural Language Generation, pages 242-249.

Robert Dale, Ilya Anisimoff, and George Narroway. 2012. HOO 2012: A report on the preposition and determiner error correction shared task. In Proceedings of the 7th Workshop on the Innovative Use of NLP for Building Educational Applications, pages 54-62.

\footnotetext{
${ }^{4}$ http://www.comp.nus.edu.sg/ nlp/conll13st.html
}

Marie-Catherine de Marneffe, Bill MacCartney, and Christopher D. Manning. 2006. Generating typed dependency parses from phrase structure parses. In Proceedings of the Fifth Conference on Language Resources and Evaluation, pages 449-454.

Michael Gamon. 2010. Using mostly native data to correct errors in learners' writing: A meta-classifier approach. In Proceedings of the Annual Meeting of the North American Chapter of the Association for Computational Linguistics, pages 163-171.

Na-Rae Han, Martin Chodorow, and Claudia Leacock. 2006. Detecting errors in English article usage by non-native speakers. Natural Language Engineering, 12(2):115-129.

Dan Klein and Christopher D. Manning. 2003. Accurate unlexicalized parsing. In Proceedings of the 41st Annual Meeting of the Association for Computational Linguistics, pages 423-430.

Claudia Leacock, Martin Chodorow, Michael Gamon, and Joel Tetreault. 2010. Automated Grammatical Error Detection for Language Learners. Morgan \& Claypool Publishers.

Diane Nicholls. 2003. The Cambridge Learner Corpus: Error coding and analysis for lexicography and ELT. In Proceedings of the Corpus Linguistics 2003 Conference, pages 572-581.

Alla Rozovskaya and Dan Roth. 2010. Generating confusion sets for context-sensitive error correction. In Proceedings of the 2010 Conference on Empirical Methods in Natural Language Processing, pages 961-970.

Alla Rozovskaya, Kai-Wei Chang, Mark Sammons, and Dan Roth. 2013. The University of Illinois system in the CoNLL-2013 shared task. In Proceedings of the Seventeenth Conference on Computational Natural Language Learning: Shared Task.

Joel Tetreault, Jennifer Foster, and Martin Chodorow. 2010. Using parse features for preposition selection and error detection. In Proceedings of the ACL 2010 Conference Short Papers, pages 353-358.

Helen Yannakoudakis, Ted Briscoe, and Ben Medlock. 2011. A new dataset and method for automatically grading ESOL texts. In Proceedings of the 49th Annual Meeting of the Association for Computational Linguistics, pages 180-189.

Ippei Yoshimoto, Tomoya Kose, Kensuke Mitsuzawa, Keisuke Sakaguchi, Tomoya Mizumoto, Yuta Hayashibe, Mamoru Komachi, and Yuji Matsumoto. 2013. NAIST at 2013 CoNLL grammatical error correction shared task. In Proceedings of the Seventeenth Conference on Computational Natural Language Learning: Shared Task. 